\title{
A Phase II Trial of Cabozantinib in Hormone Receptor-Positive Breast Cancer with Bone Metastases
}

Jing Xu, ${ }^{a, c,+}$ Michaela J. Higgins, ${ }^{d,+}$ Sara M. Tolaney, ${ }^{c, e}$ Steven E. Come, ${ }^{c, f}$ Matthew R. Smith, ${ }^{a, c}$ Monica Fornier, ${ }^{\text {g }}$ Umar Mahmood, ${ }^{\text {b,c }}$ Jose Baselga, ${ }^{\text {g,h }}$ Beow Y. Yeap, ${ }^{\text {a,c }}$ Bruce A. Chabner, ${ }^{\text {a,c }}$ Steven J. Isakoff ${ }^{a, c}$

${ }^{a}$ Massachusetts General Hospital Cancer Center, Boston, Massachusetts, USA; ${ }^{b}$ Department of Radiology, Massachusetts General Hospital, Boston, Massachusetts, USA; ${ }^{~}$ Harvard Medical School, Boston, Massachusetts, USA; ${ }^{d}$ Department of Medical Oncology, Mater Misericordiae University Hospital, Dublin, Ireland; 'Department of Medical Oncology, Dana Farber Cancer Institute, Boston,

Massachusetts, USA; ${ }^{\mathrm{f} D e p a r t m e n t}$ of Medical Oncology, Beth Israel Deaconess Medical Center, Boston, Massachusetts, USA; ${ }^{\mathrm{g}}$ Memorial Sloan Kettering Cancer Center, New York, New York, USA; ${ }^{h}$ Research \& Development Oncology, AstraZeneca Pharmaceuticals,

Gaithersburg, Maryland, USA

${ }^{\dagger}$ Contributed equally.

Disclosures of potential conflicts of interest may be found at the end of this article.

Key Words. Hormone receptor-positive breast cancer • Cabozantinib • Bone scan response - Metastatic breast cancer • Bone metastases

\section{Abstract}

Background. We assessed the antitumor activity of cabozantinib, a potent multireceptor oral tyrosine kinase inhibitor, in patients with hormone receptor-positive breast cancer with bone metastases.

Patients and Methods. In this single-arm multicenter phase II study, patients received an initial starting dose of $100 \mathrm{mg}$, later reduced to $60 \mathrm{mg}$, per day. The primary endpoint was the bone scan response rate. Secondary endpoints included objective response rate by RECIST, progression-free survival (PFS), and overall survival (OS).

Results. Of 52 women enrolled, 20 (38\%) experienced a partial response on bone scan and $6(12 \%)$ had stable disease. Prior to the first repeat bone scan at 12 weeks, 19 (35\%) patients discontinued study treatment because of early clinical progression or unacceptable toxicity. RECIST evaluation based on best overall response by computed tomography revealed stable disease in extraosseous tissues in 26 patients (50\%) but no complete or partial responses. In 25 patients with disease control on bone scan at 12 weeks, only $3(12 \%)$ patients developed extraosseous progression. The median PFS was 4.3 months, and median OS was 19.6 months. The most common grade 3 or 4 toxicities were hypertension (10\%), anorexia (6\%), diarrhea (6\%), fatigue (4\%), and hypophosphatemia (4\%).

Conclusion. Bone scans improved in $38 \%$ of patients with metastatic hormone receptor-positive breast cancer and remained stable in an additional $12 \%$ for a minimum duration of 12 weeks on cabozantinib. Further investigations should assess the activity of cabozantinib in combination with other hormonal and other breast cancer therapies and determine whether bone scan responses correlate with meaningful antitumor effects. ClinicalTrials.gov identifier. NCT01441947 The Oncologist 2020;25:652-660

Implications for Practice: Most patients with metastatic hormone receptor-positive (HR+) breast cancer have bone involvement, and many have bone-only disease, which is difficult to evaluate for response. This phase II single-arm study evaluated the clinical activity of the small molecule MET/RET/VEGFR2 inhibitor cabozantinib in patients with metastatic HR+ breast cancer with bone metastases. This study met its primary endpoint, and cabozantinib treatment resulted in a significant bone scan response rate correlating with improved survival. This is the first study to use bone scan response as a primary endpoint in breast cancer. The results support further study of cabozantinib in HR+ breast cancer.

Correspondence: Steven J. Isakoff, M.D. Ph.D., Massachusetts General Hospital Cancer Center, 55 Fruit St., Boston, Massachusetts 02114, USA. Telephone: 617-726-4920; e-mail: sisakoff@mgh.harvard.edu Received February 18, 2020; accepted for publication April 29, 2020; published Online First on June 18, 2020. http://dx.doi.org/10.1634/theoncologist.2020-0127

This is an open access article under the terms of the Creative Commons Attribution-NonCommercial-NoDerivs License, which permits use and distribution in any medium, provided the original work is properly cited, the use is non-commercial and no modifications or adaptations are made. 


\section{INTRODUCTION}

Cabozantinib (Cabometyx, XL184; Exelixis, Alameda, CA) inhibits multiple receptor tyrosine kinases, including MET, RET, vascular endothelial growth factor receptor 2 (VEGFR2), and AXL [1-3]. These kinases play an important role in tumor angiogenesis, invasion, and metastasis [1-3]. Cabozantinib is approved for the treatment of metastatic medullary thyroid cancer, advanced renal cell carcinoma, and hepatocellular carcinoma [4-6].

Overexpression of MET has been observed in all pathological subtypes of breast cancers [1]. Both MET and its ligand hepatocyte growth factor promote tumor proliferation, angiogenesis, progression, and survival $[7,8]$. RET overexpression in hormone receptor-positive ( $\mathrm{HR}+)$ breast cancer is also associated with endocrine resistance to tamoxifen and aromatase inhibitors [9-11]. A recent study of metastatic breast cancer discovered RET alterations, including amplification, missense mutations, and fusions, which activate RET kinase and downstream signaling pathways through MAPK and PI3K [2]. Furthermore, cabozantinib showed antitumor activity in a patient with RET fusion-positive tumor in the same study. High expression of VEGFR2 in breast cancer may promote tumor progression and metastasis [3, 12]. Based on these considerations, cabozantinib offers a promising therapeutic strategy for breast cancer.

Currently, clinical experience with cabozantinib in breast cancer is limited. in vitro and in vivo studies have shown that cabozantinib inhibits MET, VEGFR2, and RET, resulting in antitumor activity in many tumor models, including breast cancer [13-17]. In a phase II clinical study, 45 patients with metastatic estrogen receptor-positive $(E R+)$ or triple-negative breast cancer (TNBC) received cabozantinib, and 21 (47\%) achieved partial response or stable disease [18]. Overexpression of MET is associated with poor clinical outcomes in TNBC, and cabozantinib showed a clinical benefit rate of $34 \%$ in this subset of patients after 15 weeks of treatment [19]. Further investigations are required to establish the activity of cabozantinib in breast cancer subgroups and identify biomarkers of response.

Up to $75 \%$ of patients with metastatic breast cancer develop bone metastases during the course of their disease with even higher rates in hormone receptor-positive disease [20-22]. For many patients with ER+ metastatic breast cancer, bone may be their only metastatic site [23]. Metastatic bone lesions from cancer as imaged on a bone scan, fluorodeoxyglucose (FDG)-positron emission tomography (PET) scan, or plain films are not considered "measurable" by the widely accepted RECIST version 1.1 [24]. This excludes many patients with bone-only disease from the many clinical trials that require response measurements as a primary endpoint. Therefore, there remains a need to optimize a clinical trial endpoint in this subpopulation and to evaluate novel agents that may effectively treat their metastatic disease burden in bone as well as soft tissue sites.

In a previous phase III trial of cabozantinib in metastatic prostate cancer, bone scan response (BSR) at week 12 was used as a key secondary end point [25]. Interestingly, in this study cabozantinib produced a $42 \%$ BSR at week 12 . However, the benefit in bone scan response did not translate to a significant improvement in overall survival (OS) [25]. The lack of correlation of bone scan improvement with improved overall survival led to speculation that the drug, perhaps through its antiangiogenic activity, is limiting bone scan nuclide uptake.

This trial sought to evaluate the BSR to cabozantinib in patients with metastatic breast cancer and whether BSR may be a reliable surrogate primary endpoint for determining clinical benefit. We report an open-label, single-arm, multisite phase II trial of cabozantinib in women with metastatic HR+ breast cancer with bone metastases. The primary endpoint of this study was bone scan response rate, and we also evaluated whether bone scan response correlated with improved survival.

\section{Materials ANd Methods}

\section{Patients}

Eligible patients were $\geq 18$ years of age and had histologically confirmed ER+ and/or progesterone receptor-positive (PgR+), HER2-negative metastatic breast cancer with clear evidence of metastases to bone on isotope bone scan at screening, with or without extraosseous metastases. At baseline, subjects with bone-only disease were required to have at least two bone lesions that were not within a previously irradiated field. Patients had an Eastern Cooperative Oncology Group performance status of $<2$, an estimated life expectancy of $>3$ months, at least one prior line of hormonal or chemotherapy for treatment of their metastatic disease, and adequate liver, kidney, and marrow function. Patients were excluded if they had received chemotherapy, bone modulating agents, small-molecular kinase inhibitors or any investigational agent within 4 weeks, or hormonal anticancer therapy, immunotherapy, or radiotherapy to bone and brain metastasis within 2 weeks, or biological agents within 6 weeks before the first dose of cabozantinib.

The study protocol and informed consent documents were reviewed and approved by the institutional review boards at Dana Farber Cancer Institute (DFCI) and Memorial Sloan-Kettering Cancer Center (MSKCC), and all patients provided written informed consent. This study was performed according to the Declaration of Helsinki and International Conference on Harmonization Good Clinical Practice guidelines.

\section{Study Design and Treatment}

This open-label, multicenter, single-arm study was conducted at Massachusetts General Hospital, DFCl, Beth Israel Deaconess Medical Center, and MSKCC. The primary endpoint was bone scan response rate. Secondary endpoints included objective response rate of extraosseous sites by RECIST and overall and progression-free survival.

Patients received cabozantinib orally at 100 or $60 \mathrm{mg}$ (freebase weight) once per day. The initial dose was reduced from 100 to $60 \mathrm{mg}$ after enrollment of the first seven patients because of excessive toxicity at the higher dose. All patients were instructed to take cabozantinib each morning in a fasting state either 2 hours after or 1 hour before a meal and continued to take cabozantinib until disease progression or unacceptable adverse events. 


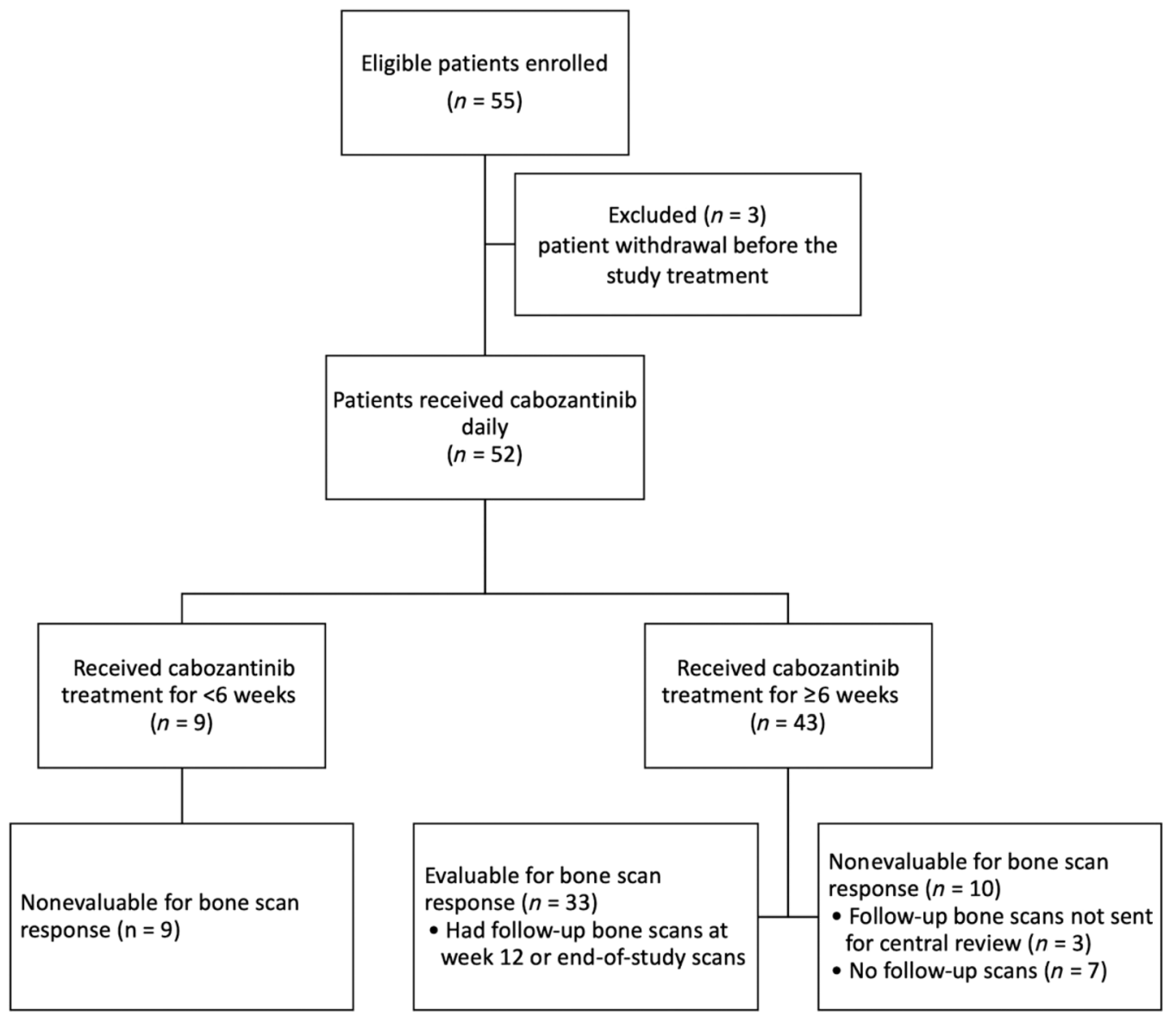

Figure 1. Flowchart of patient enrollment, treatment, and follow-up for bone scan response.

Dose reductions or interruptions were allowed for unacceptable adverse events (AEs). Cabozantinib dose was sequentially reduced from 100 to $60 \mathrm{mg}$, and in the presence of grade 3 or 4 toxicity, further reductions to $40 \mathrm{mg}$ and $20 \mathrm{mg}$ were allowed. Patients recovering from AEs within 6 weeks after dose interruptions or reductions were allowed to continue the study treatment.

\section{Study Outcomes and Assessment}

Tumor response was assessed by isotope bone scan and whole body FDG-PET/computed tomography (CT). Patients had screening bone scan and PET/CT scan within 28 days prior to the initial dose of cabozantinib and every 12 weeks thereafter until discontinuation of treatment or death. All bone and PET/CT images collected at the above prespecified times underwent independent radiologic assessment (MedQIA, Los Angeles, CA). Only patients who had at least 6 weeks of treatment and had follow-up bone scans or PET/CT scans were evaluable for response. BSR was determined by the percentage change of bone scan area from baseline. Responses were categorized as complete (complete resolution), partial ( $\geq 30 \%$ reduction), stable disease (between $<30 \%$ reduction and $<20 \%$ increase), or progressive disease ( $\geq 20 \%$ increase). Bone scan response rate was defined as the percentage of patients experiencing a complete or partial response in bone scan lesions. Overall response rate (ORR) was defined as the proportion of treated patients experiencing a complete response (CR) or a partial response (PR) as defined by RECIST version 1.1. Those who had less than 6 weeks of treatment, or had developed progressive disease or died before the reevaluation date, or had not undergone reevaluation were considered nonresponders. The best overall response was defined as the best response from the time of enrollment until termination of study treatment.

AEs were assessed from the start of treatment according to the National Cancer Institute Common Terminology Criteria for Adverse Events version 4.0.

\section{Statistical Analysis}

The primary endpoint was bone scan response rate. A total sample size of 50 was estimated using Simon's two-stage design with $92 \%$ power to detect a bone response rate of $30 \%$, compared with a null response rate of $10 \%$, at a significance level of .05 (one-sided). In the first stage, 17 patients were recruited, and if two or fewer bone responses were observed the study would be stopped. Otherwise, 33 additional patients would be accrued for a total of 50. If eight or more responses were observed in 50 patients, the null hypothesis would be rejected, and cabozantinib was considered promising for further investigation.

Descriptive statistics were used to summarize baseline characteristics. Bone scan response rate and ORR were 
reported as point estimates with $90 \%$ confidence intervals (Cls). Median OS and progression-free survival (PFS) were estimated for the overall study population, along with $90 \%$ confidence intervals, using the Kaplan-Meier approach. Stratified OS and PFS analyses were performed to compare subjects with bone lesions only with those with bone plus other site lesions to provide point estimates with $90 \%$ confidence intervals. The log-rank test was used to compare these two subgroups. Exploratory landmark analysis of OS or PFS was performed based on the 12-week bone scan responses [26]. Patients who achieved PR or stable disease (SD) on bone scans at 12 weeks were classified as the disease control (DC) group. Those who had progressive disease (PD) on bone scans or were nonevaluable at 12 weeks (because of treatment discontinuation or early disease progression) were classified as the non-DC group. In this analysis, only patients alive or progression free at the landmark time (12 weeks from baseline) were included in the analysis of overall survival or progression-free survival. Kaplan-Meier estimates of survival probability were conditional on the status of bone scan response at the landmark time of 12 weeks for patients who survived up to this time. Treatment-related grade 3 or 4 were summarized by descriptive statistics.

\section{RESULTS}

\section{Patients and Treatment}

From November 2011 through January 2013, a total of 55 patients were enrolled at four study centers. Three patients withdrew from this study before receiving any treatment. In total, 52 patients received cabozantinib treatment and were evaluable for data analysis. The flowchart (Fig. 1) shows the status of patient enrollment, treatment, and follow-up for the primary endpoint. In the first stage, 4 of 17 patients showed significant improvement (PR) after the 12-week bone scan, and the study continued to stage 2 .

Baseline demographics and clinical characteristics of the 52 treated patients are listed in Table 1 (and supplemental online Tables 1 and 2). All patients were ER+, as assessed in either metastatic lesions (45 patients) or primary tissues (7 patients), and 40 patients (77\%) were PgR+. All patients had evidence of bone metastases on bone scan at baseline and 18 (35\%) had bone-only disease. The majority of patients were heavily pretreated for metastatic disease, with $45(87 \%)$ and 37 patients (71\%) having previously received endocrine therapy or chemotherapy, respectively. Twentyeight (54\%) and 22 patients (42\%) had at least two lines of endocrine therapy or chemotherapy, respectively, for metastatic disease, and 35 patients (67\%) had received bisphosphonate bone modifying agents.

The first seven patients (13\%) had an initial cabozantinib daily dose of $100 \mathrm{mg}$ (freebase weight), but because of toxicity the remaining patients started at a daily dose of $60 \mathrm{mg}$. Treatment durations of patients ranged from 1 to 204 weeks; 30 patients were treated for at least 12 weeks, and 22 were treated for less than 12 weeks, of whom 9 had less than 6 weeks of treatment.
Table 1. Baseline characteristics of patients

\begin{tabular}{ll}
\hline Characteristics & $\begin{array}{l}\text { Summary statistics } \\
(\boldsymbol{n}=\mathbf{5 2}), \boldsymbol{n}(\%)\end{array}$ \\
\hline Female sex & $52(100)$ \\
Median age (range), years & $55(33-79)$ \\
White race & $42(81)$ \\
ECOG performance status & \\
0 & $37(71)$ \\
1 & $15(29)$ \\
Estrogen $/$ progesterone receptors & \\
ER+/PgR+ & $40(77)$ \\
ER+/PgR- & $12(23)$ \\
Sites of disease & \\
Bone only & $18(35)$ \\
Bone + others & $34(65)$ \\
Liver & $5(10)$ \\
Lung/pleura & $7(13)$ \\
Brain & 0 \\
One other site & $8(15)$ \\
$\geq 2$ other sites & $14(27)$ \\
Prior lines of endocrine therapy for & \\
metastatic disease & \\
0 & $28(54)$ \\
1 & \\
$2-4$ & \\
Prior & \\
\hline
\end{tabular}

Prior lines of chemotherapy for metastatic disease

0

1

2-9

Prior therapy of bone modifying agents

Bisphosphonates

Denosumab

${ }^{\mathrm{a}} \mathrm{ER}+$ in either metastatic lesions (45 patients) or primary tissues (7 patients).

Abbreviations: ECOG, Eastern Cooperative Oncology Group; ER+, estrogen receptor positive; PgR, progesterone receptor.

\section{Bone Scan Response}

All 52 patients who received cabozantinib treatment were included in the data analysis for bone scan responses. According to the prespecified criteria, 19 patients (36.5\%) were considered nonevaluable for bone scan response because either they had no follow-up images ( $n=16$, early treatment discontinuation before reevaluation date) or follow-up images were not available for central review $(n=3)$. The remaining 33 patients $(63.5 \%)$ who received at least 6 weeks of cabozantinib and had at least one followup bone scan at week 12 or at the last study point were considered evaluable for bone scan responses.

Table 2 shows the bone scan responses to cabozantinib treatment. The bone scan response rate was $38.5 \%(20 / 52$; $90 \% \mathrm{Cl}, 27.1 \%-51.0 \%)$, including 20 patients who achieved 
A

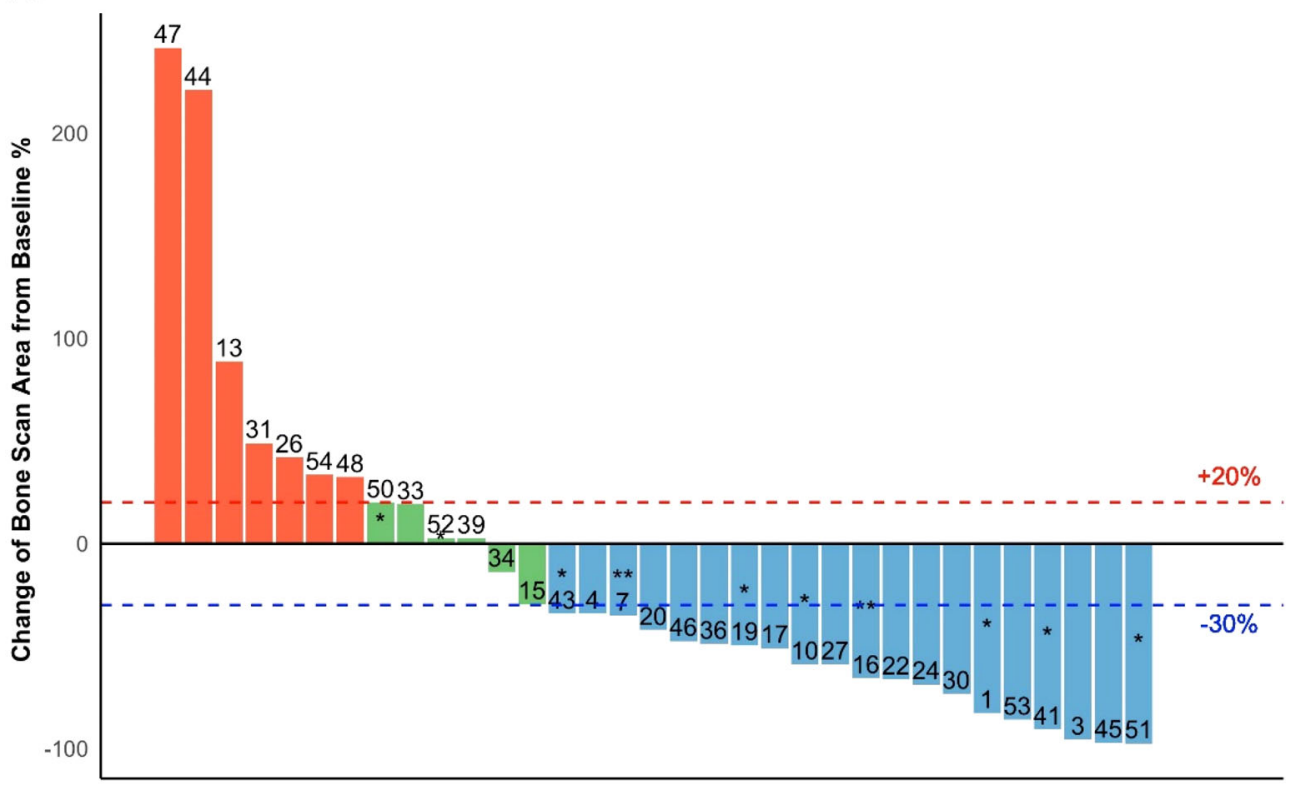

Best overall response

Partial response

Stable disease

Progressive disease

Patient (ID)

B

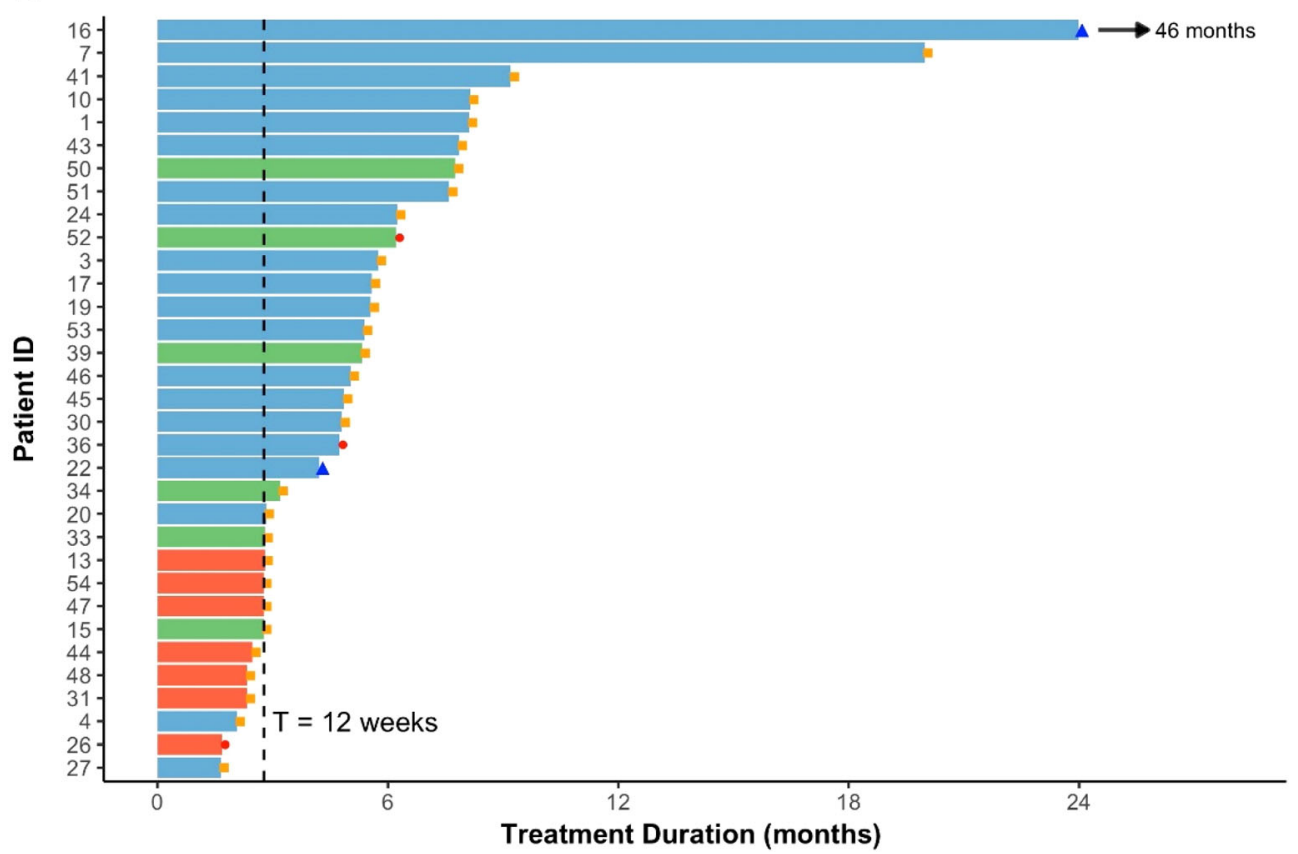

Response type

Partial repsonse

Stable disease

Progressive disease

Off treatment reason

- Progression

- Toxicity

- Others

Figure 2. Best overall bone scan response among 33 patients with follow-up bone scan. (A): Waterfall plot of best overall bone scan response. *, treatment duration over 6 months; **, treatment duration over 12 months. (B): Swimmer plot of treatment duration with best overall bone scan response. Patients 16 and 22 discontinued treatment because of physician discretion and impending pathological fracture, respectively.

a PR and none with a CR of bone lesions. The disease control rate (DCR) was $50 \%$ (26/52), defined as the percentage of patients with PR or SD (six patients), based on the best overall response by bone scan. Progressive disease was the best response in seven patients (13.5\%). Among 18 patients with bone-only disease, the bone scan response rate was $61 \%$, and in 34 patents with extraosseous disease, the bone scan response rate was $26.5 \%$ (Table $2 \mathrm{~B}$ ).

Figure $2 \mathrm{~A}$ shows the waterfall plot of best overall bone scan response for the patients having a 12-week follow-up bone scan $(n=33)$. Of these patients, $60.6 \%(20 / 33)$ demonstrated at least $30 \%$ reduction in bone scan lesion area, and $78.8 \%(26 / 33)$ achieved disease control (PR + SD); $30.3 \%(10 / 33)$ had treatment duration for over 6 months, and their bone scans demonstrated either PR or SD. Two patients received treatment for over 12 months and achieved significant improvement (PR) in bone lesions. Figure $2 \mathrm{~B}$ shows the treatment duration with bone scan response for the 33 protocol-defined evaluable patients who had baseline and repeated bone scans. Among the 
Table 2. Response to treatment

\begin{tabular}{lcc}
\hline \multicolumn{3}{c}{ (A): Bone scan response and RECIST response } \\
\hline & \multicolumn{2}{c}{ Method of response assessment } \\
\cline { 2 - 3 } Response $^{\text {bone scan }}$ & $(\boldsymbol{n}=\mathbf{5 2}), \boldsymbol{n}(\%)$ & $\begin{array}{l}\text { RECIST } \\
(\boldsymbol{n}=\mathbf{5 2}), \boldsymbol{n}(\%)\end{array}$ \\
\hline CR & 0 & 0 \\
PR & $20(38.5)$ & 0 \\
SD & $6(11.5)$ & $26(50.0)$ \\
PD & $7(13.5)$ & $7(13.5)$ \\
Nonevaluable & $19(36.5)$ & $19(36.5)$ \\
Bone scan response & $38.5(27.1-51.0)$ & \\
rate (90\% Cl), \% & & 0 \\
Overall response rate & & \\
\hline
\end{tabular}

(B): Subgroup analysis of bone scan response by disease site

\begin{tabular}{lcc}
\hline & \multicolumn{2}{c}{ Disease sites at baseline } \\
\cline { 2 - 3 } Response & $\begin{array}{l}\text { Bone only } \\
(\boldsymbol{n}=\mathbf{1 8}), \boldsymbol{n}(\%)\end{array}$ & $\begin{array}{l}\text { Bone }+ \text { other } \\
(\boldsymbol{n}=\mathbf{3 4 )}, \boldsymbol{n}(\%)\end{array}$ \\
\hline CR & $0(0)$ & $0(0)$ \\
PR & $11(61.1)$ & $9(26.5)$ \\
SD & $0(0)$ & $6(17.6)$ \\
PD & $2(11.1)$ & $5(14.7)$ \\
Nonevaluable $^{c}$ & $5(27.8)$ & $14(41.2)$ \\
\hline
\end{tabular}

\begin{abstract}
${ }^{a}$ All responses are based on best overall response during the study period. The percentage calculations are based on intention-to-treat analysis using a denominator of 52 patients who received cabozantinib treatment in this trial.

${ }^{\mathrm{b}}$ Patients evaluable for bone scan response are defined as patients who received $\geq 6$ weeks of cabozantinib and had at least one follow-up bone scan evaluable for central review. Patients evaluable for RECIST response are defined as patients who received $\geq 6$ weeks of cabozantinib and had at least one follow-up computed tomography scan evaluable for central review.

'The 19 patients nonevaluable for bone scan response include 16 patients who discontinued treatment prior to reevaluation date because of toxicity (4), disease progression (9), or patient withdrawal from trial including too ill to continue (1), difficulty traveling (1), and unrelated illness (1). An additional three patients did not have their bone scans sent for central review. All protocol-defined nonevaluable patients are included in the intention-to-treat analysis.

Abbreviations: $\mathrm{Cl}$, confidence interval; $\mathrm{CR}$, complete response; $\mathrm{PR}$, partial response; SD, stable disease; PD, progressive disease.
\end{abstract}

24 patients who had cabozantinib treatment of 12 weeks duration or longer, $96 \%$ achieved disease control (PR or SD) as assessed by bone lesions, and one patient received cabozantinib treatment for 46 months. Patients discontinued treatment because of progression $(n=28)$, unacceptable toxicity $(n=3)$, and other reasons including impending pathological fracture $(n=1)$ and physician discretion $(n=1)$.

\section{ORR by RECIST}

The ORR was evaluated based on the results of CT scans by conventional RECIST criteria. As shown in Table 2, 33 of 52 patients had at least one follow-up evaluation for ORR per RECIST. Those without subsequent evaluation were treated as nonresponders $(n=19)$. No patients achieved a CR or PR per RECIST, 26 (50\%) achieved SD, and 7 (13.5\%) had PD at extraosseous sites.

The correlation between bone scan response and RECIST response at 12 weeks was further explored (Table 3 and supplemental online Fig. 1). Thirty-one of 33 patients who
Table 3. Correlation of bone scan and RECIST response at 12 weeks $(n=33)^{\text {a }}$

\begin{tabular}{llll}
\hline & \multicolumn{3}{c}{ By RECIST } \\
\cline { 2 - 4 } By bone scan & SD, $\boldsymbol{n}(\%)$ & PD, $\boldsymbol{n}(\%)$ & UE, $\boldsymbol{n}(\%)$ \\
\hline PR & $12(85.7)$ & $1(7.1)$ & $1(7.1)^{\mathrm{b}}$ \\
SD & $8(72.7)$ & $2(18.2)$ & $1(9.1)^{\mathrm{b}}$ \\
PD & $5(62.5)$ & $3(37.5)$ & $0(0)$ \\
\hline
\end{tabular}

${ }^{a}$ Thirty-three patients were evaluable for bone scan response by central review at 12 weeks. Thirty-one of them had their RECIST response evaluated by central review at 12 weeks.

${ }^{\mathrm{b}}$ Two patients had their RECIST response unevaluable by central review at 12 weeks, but institutional computed tomography scans showed stable disease at extraosseous sites for these two patients at 12 and 18 weeks, respectively.

Abbreviations: PR, partial response; SD, stable disease; PD, progressive disease; UE, unable to evaluate.

were evaluable for bone scan response at 12 weeks also had their RECIST response evaluated by central review at the same time. Among 25 patients who had disease control $(P R+S D)$ on bone scan at 12 weeks, none achieved a RECIST partial response, 20 patients (80\%) had stable disease, 3 patients (12\%) had progressive disease, and the other 2 patients (8\%) were unevaluable for RECIST. Of eight patients who had PD on bone scan at 12 weeks, three patients (37.5\%) had RECIST PD, and five patients (62.5\%) had SD in extraosseous lesions.

\section{Overall Survival/Progression-Free Survival}

Fifty-two patients were followed for over 5 years (Fig. 3A and B). The median follow-up time was 28.5 months (1.5-86.3 months). The median OS was 19.6 months $(90 \% \mathrm{Cl}$, 18.0-26.8 months), and median PFS was 4.3 months ( $90 \%$ $\mathrm{Cl}, 2.8-5.5$ months).

Both OS and PFS analyses were stratified based on metastatic sites (bone only vs. bone plus other sites) at baseline. The stratified median OS was 26.8 months ( $90 \% \mathrm{Cl}, 21.1-33.0$ months) for patients with bone only lesions and 18.7 months (90\% Cl, 14.3-20.0 months) for those with bone and other lesions (hazard ratio, $0.64 ; 90 \% \mathrm{Cl}, 0.37-1.1 ; p=.17$; Fig. $3 \mathrm{C}$ ). The median PFS was 4.9 months $(90 \% \mathrm{Cl}, 2.8-7.8$ months) for patients with bone-only lesions and 3.4 months $(90 \% \mathrm{Cl}$, 2.7-5.8 months) for those with bone plus other lesions (hazard radio, $0.91 ; 90 \% \mathrm{Cl}, 0.56-1.5 ; p=.77$; supplemental online Fig. 2). No significant difference in OS and PFS was observed among patients with bone only versus bone plus other sites.

In a post hoc analysis, the landmark method was used to determine whether the bone scan responses correlated with improved survival. The landmark estimates were based on the bone scan status at 12 weeks. The 48 patients known to be alive at 12 weeks were included for OS analysis (Fig. 3D), and the 31 patients alive without death or progression at 12 weeks were included in the PFS analysis (supplemental online Fig. 3). A significant difference was observed between patients with and without disease control by bone scan response (Fig. 3D). The group with disease control at 12 weeks had significantly longer survival (median OS, 24.2 months; 90\% Cl, 16.4-31.7 months) compared with that without disease control (median OS, 13.3 months; $90 \% \mathrm{Cl}$, 9.5-18.2 months) with a hazard ratio of $0.37(90 \% \mathrm{Cl}, 0.21-0.65)$. No PFS benefit was observed for 
A

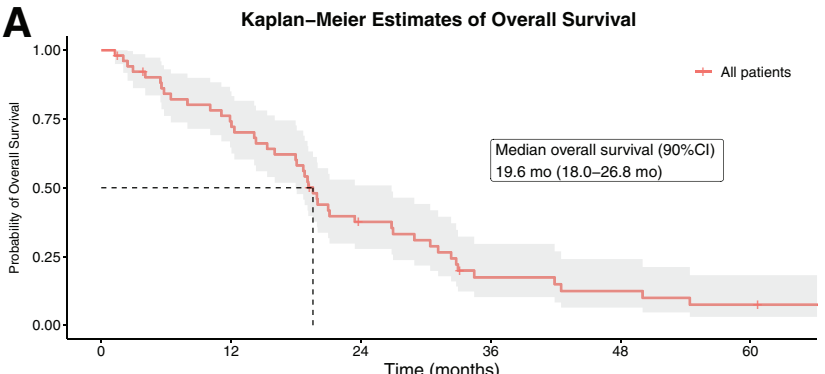

Number at risk with time

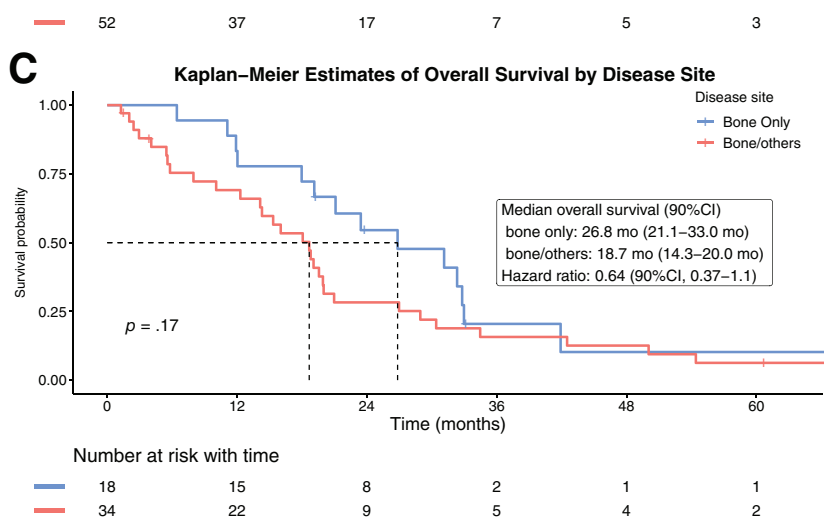

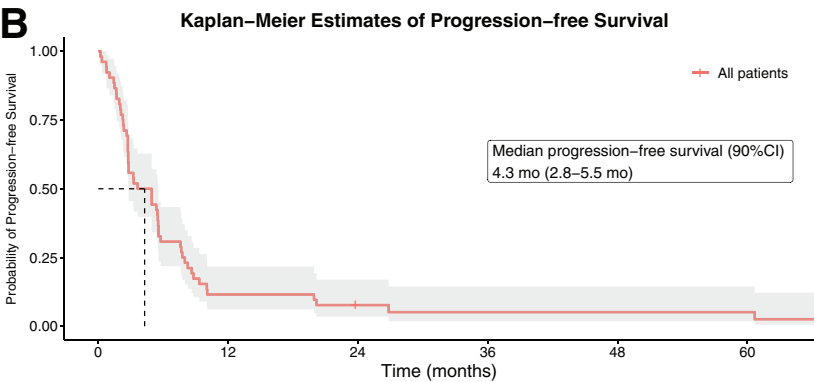

Number at risk with time

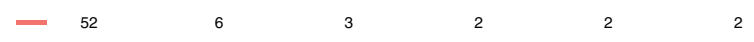

D Landmark Analysis of Overall Survival by Disease Control Status at 12 Weeks

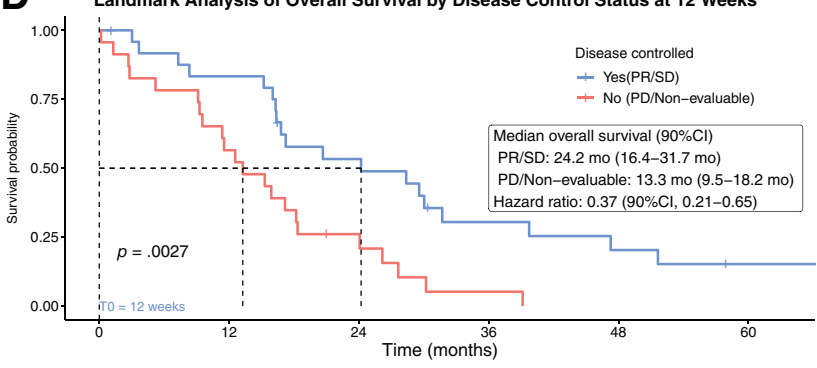
Number at risk with time

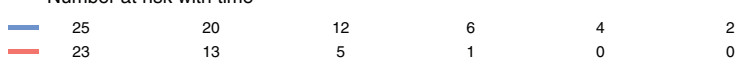

Figure 3. Kaplan-Meier estimates of overall survival, progression-free survival, and overall survival according to subgroups. KaplanMeier estimates of overall survival (A), progression-free survival (B), overall survival by different disease site at baseline (C), and landmark analysis of overall survival by disease control status based on bone scan response at 12 weeks (D); in panel D, time zero started after 12 weeks of treatment, and three patients died and one was censored before 12 weeks; thus 48 patients were included in landmark overall survival analysis.

Abbreviations: $\mathrm{Cl}$, confidence interval; $\mathrm{PD}$, progressive disease; PR, partial response; $\mathrm{SD}$, stable disease.

the disease control group (supplemental online Fig. 3), but the number of patients at risk in each subset was small ( 9 in nondisease control group vs. 22 in disease control group).

\section{Adverse Events}

All 52 patients who received cabozantinib treatment are included in the safety analysis. The median treatment duration was 12 weeks (range, 1-204 weeks). Patients received a median average daily dose of $51.5 \mathrm{mg}$ (range, 21.4-100 $\mathrm{mg})$. Forty-two patients $(80.8 \%)$ had dose modifications (dose reduction or dose held), and seven patients (13.5\%), who all started at the $60 \mathrm{mg}$ dose, discontinued their treatments because of unacceptable toxicity.

Of the 52 patients, three had received treatments for less than 3 weeks (one cycle), and no related adverse events were reported for these patients. In total, 123 types of related AEs were reported for 49 patients (94.2\%). The most common allgrade AEs were fatigue (63\%), elevation in either alanine aminotransferase $(52 \%)$ or aspartate aminotransferase $(48 \%)$, diarrhea (48\%), nausea (46\%), palmar-plantar erythrodysesthesia syndrome (44\%), oral mucositis (37\%), and anorexia (35\%). A total of 19 grade 3 AEs was observed in 26 patients. The most frequent grade 3 AEs were hypertension $(10 \%)$, anorexia (6\%), diarrhea (6\%), fatigue (4\%), hypophosphatemia (4\%), lymphocyte count decrease (4\%), febrile neutropenia (4\%), and hyponatremia (4\%; Table 4). Three grade 4 AEs were seen in three patients, including dehydration, hypercalcemia, and neutropenia. No deaths occurred during the treatment period of this study. The common AEs leading to treatment discontinuation included fatigue, dyspnea, anorexia, diarrhea, hypertension, and musculoskeletal and connective tissue disorder.

\section{Discussion}

In this single-arm phase II study in patients with hormone receptor-positive breast cancer with bone metastases, cabozantinib treatment resulted in a high rate of bone scan response and disease control rate. Patients who achieved disease control on bone scan to cabozantinib had improved overall survival compared with patients without disease control. Responses assessed by traditional RECIST were not observed, but prolonged disease control using traditional response evaluation by $\mathrm{CT}$ imaging was observed in a significant percentage of patients. Together, our findings suggest that cabozantinib is active in advanced HR+ breast cancer with bone metastases.

To our knowledge, this is the first study to use prespecified bone scan response in breast cancer as the primary endpoint. In this study, bone scan response correlated with overall survival and disease control in extraosseous sites. Interestingly, $80 \%$ of patients who achieved disease control with bone scans at 12 weeks maintained the same status in extraosseous lesions. This suggests that the bone scan responses may reflect antitumor activity in HR+ metastatic breast cancer. Furthermore, in a landmark analysis, patients with disease control $(P R+S D)$ based on the bone scan response at 12 weeks had a longer survival compared with those in the non-disease control group (PD + nonevaluable). These findings support the notion that disease control status based on 12-week bone 
Table 4. Treatment-related grade 3 or 4 adverse events

\begin{tabular}{lll}
\hline Adverse event & $\begin{array}{l}\text { Grade 3, } \\
\boldsymbol{n}(\%)\end{array}$ & $\begin{array}{l}\text { Grade 4, } \\
\boldsymbol{n}(\%)\end{array}$ \\
\hline Hypertension & $5(10)$ & 0 \\
Anorexia & $3(6)$ & 0 \\
Diarrhea & $3(6)$ & 0 \\
Fatigue & $2(4)$ & 0 \\
Febrile neutropenia & $2(4)$ & 0 \\
Hyponatremia & $2(4)$ & 0 \\
Hypophosphatemia & $2(4)$ & 0 \\
Lymphocyte count decreased & $2(4)$ & 0 \\
Alanine aminotransferase & $1(2)$ & 0 \\
increased & $1(2)$ & 0 \\
Alkaline phosphatase increased & $1(2)$ & 0 \\
Cardiac disorders & $1(2)$ & 0 \\
Erythema multiforme & $1(2)$ & 0 \\
Fracture & $1(2)$ & 0 \\
Hypokalemia & $1(2)$ & $1(2)$ \\
Neutropenia & $1(2)$ & 0 \\
Lung infection & $1(2)$ & 0 \\
Mucositis oral & $1(2)$ & 0 \\
Palmar-plantar & $1(2)$ & 0 \\
erythrodysesthesia syndrome & 0 & $1(2)$ \\
Pulmonary embolus & 0 & $1(2)$ \\
Dehydration & & \\
Hypercalcemia & & 0 \\
\hline
\end{tabular}

scans may predict patient survival and may guide continuation of cabozantinib treatment. Definitive proof of clinical benefit would require a randomized trial. Our primary finding is in agreement with a previous study in metastatic prostate cancer where cabozantinib improved bone scans with a BSR of $42 \%$ and a DCR of $62 \%$. The lack of correlation of bone scan findings with survival improvement in prostate cancer patients treated with cabozantinib leaves unanswered whether the bone scan changes reflect true disease control, and potential clinical benefit, or simply interference with radionuclide uptake in bone lesions [25]. However, that study was randomized and did not include a similar landmark analysis among the patients with cabozantinib-treated prostate cancer, thereby limiting direct comparison of the different results.

A challenge with cabozantinib use has been intolerance to treatment. The most common toxicities leading to discontinuation are fatigue, abnormal liver function, diarrhea, and nausea, as well as hypertension and proteinuria, which are associated with its antiangiogenic action [4-6]. In our study, cabozantinib was not tolerable at the $100 \mathrm{mg} /$ day starting dose in all patients, but at the currently approved dose of $60 \mathrm{mg} / \mathrm{day}$ it was better tolerated through dose modification and other supportive care. The rate of treatment discontinuation (13.5\%) because of unacceptable AEs was similar to the rate (16\%) observed in phase III trials of hepatocellular carcinoma and thyroid cancer $[4,6]$. Our results are similar to the $88 \%$ rate of dose reduction reported in the phase III study of metastatic castration-resistant prostate cancer in which the starting dose of $100 \mathrm{mg}$ was used [25].
The results of our study in hormone receptor-positive breast cancer, together with prior results showing a clinical benefit rate of $34 \%$ in triple-negative breast cancer, raise the possibility of testing cabozantinib in combination with other therapies. However, studies evaluating cabozantinib and nivolumab in TNBC and cabozantinib and trastuzumab in patients with breast cancer brain metastases showed insufficient activity to support further development of the combinations [27, 28]. In our study, a separate cohort combining cabozantinib with fulvestrant was initiated but closed early for slow accrual. An ongoing trial is evaluating the efficacy of cabozantinib in combination with atezolizumab for metastatic breast cancer (ClinicalTrials.gov NCT03170960).

A limitation of this study was the single-arm study design, which did not permit comparison with a standard therapy. Therefore, it was not possible to assess the clinical benefit for cabozantinib as compared with other available therapies. The small sample size precluded more detailed subgroup analysis for clinical endpoints. Furthermore, our use of bone scan response as a novel primary endpoint in this proof of concept study would not be appropriate as a single measure of efficacy in a larger study because it neglects assessment of extraosseous lesions. In addition, the identification of biomarkers that may correlate with bone scan response or extraosseous response is needed.

\section{Conclusion}

In this phase II study we showed that cabozantinib monotherapy led to improvement in bone lesions in pretreated patients with $\mathrm{HR}+$ breast cancer with bone metastases. Patients who achieved disease control by bone scan response at 12 weeks had improved overall survival. These results support further studies to evaluate the clinical activity of cabozantinib in $\mathrm{HR}+$ metastatic breast cancer and to confirm the utility of bone scan response as a meaningful tool to assess responses to treatment with this drug.

\section{ACKNOWLEDGMENTS}

This study was supported by Exelixis, Inc., which provided financial funding support and cabozantinib for this clinical trial. M.J.H. was the recipient of an ASCO Career Development Award for this trial. We thank our oncology colleagues and research staff at the participating sites for their support. We gratefully acknowledge and thank the patients and their families for participating in the study. We thank Elizabeth L. Tripp, Rachel A. Hepp, and Sara Sutherland for study coordination and data collection.

\footnotetext{
Author Contributions

Conception/design: Michaela J. Higgins, Matthew R. Smith, Umar Mahmood, Jose Baselga, Steven J. Isakoff

Provision of study material or patients: Michaela J. Higgins, Sara Tolaney, Steven E. Come, Monica Fornier, Steven J. Isakoff

Collection and/or assembly of data: Jing $\mathrm{Xu}$, Michaela J. Higgins, Bruce A. Chabner, Steven J. Isakoff

Data analysis and interpretation: Jing $\mathrm{Xu}$, Michaela J. Higgins, Sara Tolaney, Steven E. Come, Matthew R. Smith, Monica Fornier, Umar Mahmood, Jose Baselga, Beow Y. Yeap, Bruce A. Chabner, Steven J. Isakoff
} 
Manuscript writing: Jing $\mathrm{Xu}$, Michaela J. Higgins, Sara Tolaney, Steven E. Come, Matthew R. Smith, Monica Fornier, Umar Mahmood, Jose Baselga, Beow Y. Yeap, Bruce A. Chabner, Steven J. Isakoff

Final approval of manuscript: Jing $\mathrm{Xu}$, Michaela J. Higgins, Sara Tolaney, Steven E. Come, Matthew R. Smith, Monica Fornier, Umar Mahmood, Jose Baselga, Beow Y. Yeap, Bruce A. Chabner, Steven J. Isakoff

\section{Disclosures}

Michaela J. Higgins: Roche (other: travel and meeting registration);

Sara M. Tolaney: Merck, Bristol-Myers Squibb, Eli Lilly \& Co.,

Pfizer, Novartis, AstraZeneca, Eisai, Nektar, Sanofi, Immunomedics,

Genentech, Celldex, Paxman, Seattle Genetics, Nanostring, Daiichi-

Sankyo, Sanofi, Abbvie, Athenex, Puma (C/A), Exelixis, Odenate,

Cyclacel, Merck, Bristol-Myers Squibb, Eli Lilly \& Co., Pfizer,

Novartis, AstraZeneca, Eisai, Nektar, Sanofi, Immunomedics,

Genentech (RF); Matthew R. Smith: Bayer, Janssen Oncology, Eli

Lilly \& Co., Amgen, Pfizer, Novartis, Astellas Pharma (C/A), Gilead

Sciences, Bayer, Janssen Oncology, Eli Lilly \& Co. (RF); Monica

Fornier: Eisai, Genentech (C/A); Umar Mahmood: CytoSite
Biopharma (C/A, RF, OI, IP); Jose Baselga: Grail, ApoGen, Juno, Seragon, PMV Pharma, Northern Biologics, Eli Lilly \& Co., Novartis (C/A), Eli Lilly \& Co., Novartis, Roche (H), AstraZeneca (E), PMV Pharma, Varian, Foghorn Therapeutics, Aura, Infinity Pharmaceuticals, Tango, Venthera, Grail, ApoGen, Juno, Seragon (OI), Eli Lilly \& Co., Novartis, Roche (other: travel), Foghorn Therapeutics, Varian Medical Systems, Bristol-Myers Squibb, Grail, Aura Biosciences, Infinity Pharmaceuticals (other-board of directors); Bruce A. Chabner: PharmaMar, EMD Serono, Cyteir, Eli Lilly \& Co., Chugai Pharmaceuticals, Takeda (C/A), Cyteir (H), Biomarin, Seattle Genetics, GlaxoSmithKline, PharmaMar, Blueprint, Immunomedics, Constellation, Bluebird, Alnylam, SpringWorks, Forty Seven (OI), Eli Lilly \& Co., Genentech (ET); Steven J. Isakoff: Genentech, OncoPep, Abbvie, Mylan, Myriad Genetics, Immunomedics, Puma (C/A), Genentech, OncoPep, Abbvie, AstraZeneca, Genentech, Merck, Pharmamar (RF). The other authors indicated no financial relationships. (C/A) Consulting/advisory relationship; (RF) Research funding; (E) Employment; (ET) Expert testimony; (H) Honoraria received; (OI) Ownership interests; (IP) Intellectual property rights/ inventor/patent holder; (SAB) Scientific advisory board

\section{REFERENCES}

1. Raghav KP, Wang W, Liu S et al. cMET and phospho-cMET protein levels in breast cancers and survival outcomes. Clin Cancer Res 2012;18 2269-2277.

2. Paratala BS, Chung JH, Williams CB et al. RET rearrangements are actionable alterations in breast cancer. Nat Commun 2018;9:4821.

3. Yan JD, Liu Y, Zhang ZY et al. Expression and prognostic significance of VEGFR-2 in breast cancer. Pathol Res Pract 2015;211:539-543.

4. Elisei R, Schlumberger MJ, Muller SP et al. Cabozantinib in progressive medullary thyroid cancer. J Clin Oncol 2013;31:3639-3646.

5. Choueiri TK, Escudier B, Powles $T$ et al Cabozantinib versus everolimus in advanced renal-cell carcinoma. N Engl J Med 2015;373: 1814-1823.

6. Abou-Alfa GK, Meyer T, Cheng AL et al. Cabozantinib in patients with advanced and progressing hepatocellular carcinoma. N Engl J Med 2018;379:54-63.

7. Lengyel E, Prechtel D, Resau JH et al. C-Met overexpression in node-positive breast cancer identifies patients with poor clinical outcome independent of Her2/neu. Int J Cancer 2005;113. 678-682.

8. Veenstra C, Perez-Tenorio G, Stelling A et al. Met and its ligand HGF are associated with clinical outcome in breast cancer. Oncotarget 2016;7: 37145-37159.

9. Morandi A, Plaza-Menacho I, Isacke CM. RET in breast cancer: Functional and therapeutic implications. Trends Mol Med 2011;17:149-157.

10. Plaza-Menacho I, Morandi A, Robertson D et al. Targeting the receptor tyrosine kinase RET sensitizes breast cancer cells to tamoxifen treatment and reveals a role for RET in endocrine resistance. Oncogene 2010;29:4648-4657.

11. Boulay A, Breuleux M, Stephan C et al. The Ret receptor tyrosine kinase pathway functionally interacts with the ERalpha pathway in breast cancer. Cancer Res 2008;68:3743-3751.

12. Pizon M, Zimon DS, Pachmann $U$ et al. Insulin-like growth factor receptor I (IGF-IR) and vascular endothelial growth factor receptor 2 (VEGFR-2) are expressed on the circulating epithelial tumor cells of breast cancer patients. PLoS One 2013;8:e56836.

13. Yakes FM, Chen J, Tan J et al. Cabozantinib (XL184), a novel MET and VEGFR2 inhibitor, simultaneously suppresses metastasis, angiogenesis, and tumor growth. Mol Cancer Ther 2011; 10:2298-2308.

14. Sameni $M$, Tovar EA, Essenburg $\mathrm{CJ}$ et al Cabozantinib (XL184) inhibits growth and invasion of preclinical TNBC models. Clin Cancer Res 2016;22:923-934

15. Bentzien $F$, Zuzow $M$, Heald $N$ et al. In vitro and in vivo activity of cabozantinib (XL184), an inhibitor of RET, MET, and VEGFR2, in a model of medullary thyroid cancer. Thyroid 2013;23:1569-1577.

16. Zhou L, Liu XD, Sun M et al. Targeting MET and $\mathrm{AXL}$ overcomes resistance to sunitinib therapy in renal cell carcinoma. Oncogene 2016;35: 2687-2697.

17. Xiang $Q$, Chen $W$, Ren $M$ et al. Cabozantinib suppresses tumor growth and metastasis in hepatocellular carcinoma by a dual blockade of VEGFR2 and MET. Clin Cancer Res 2014;20: 2959-2970.

18. Tolaney SM, Nechushtan H, Ron IG et al. Cabozantinib for metastatic breast carcinoma: results of a phase II placebo-controlled randomized discontinuation study. Breast Cancer Res Treat 2016;160:305-312.

19. Tolaney SM, Ziehr DR, Guo $\mathrm{H}$ et al. Phase II and biomarker study of cabozantinib in metastatic triple-negative breast cancer patients. The Oncologist 2017;22:25-32.

20. Brook N, Brook E, Dharmarajan A et al. Breast cancer bone metastases: Pathogenesis and therapeutic targets. Int J Biochem Cell Biol 2018;96:63-78

21. Solomayer EF, Diel IJ, Meyberg GC et al. Metastatic breast cancer: Clinical course, prognosis and therapy related to the first site of metastasis. Breast Cancer Res Treat 2000;59:271-278.

22. Palma MA, Body JJ. Usefulness of bone formation markers in breast cancer. Int J Biol Markers 2005;20:146-155.

23. Vredenburgh JJ, Madan B, Coniglio D et al A randomized phase III comparative trial of immediate consolidation with high-dose chemotherapy and autologous peripheral blood progenitor cell support compared to observation with delayed consolidation in women with metastatic breast cancer and only bone metastases following intensive induction chemotherapy. Bone Marrow Transplant 2006;37:1009-1015

24. Eisenhauer EA, Therasse $P$, Bogaerts $J$ et al New response evaluation criteria in solid tumours: revised RECIST guideline (version 1.1) Eur J Cancer 2009;45:228-247.

25. Smith M, De Bono J, Sternberg $C$ et al. Phase III study of cabozantinib in previously treated metastatic castration-resistant prostate cancer: COMET-1. J Clin Oncol 2016;34: 3005-3013.

26. Dafni U. Landmark analysis at the 25-year landmark point. Circ Cardiovasc Qual Outcomes 2011;4:363-371.

27. Barroso-Sousa R, Trippa L, Li TY et al. A phase II study of nivolumab in combination with cabozantinib for metastatic triple-negative breast cancer (mTNBC). Cancer Res 2019;80(suppl 4): P3-09-10a.

28. Leone JP, Duda DG, Hu J et al. A phase I study of cabozantinib alone or in combination with trastuzumab in breast cancer patients with brain metastases. Breast Cancer Res Treat 2020; 179:113-123.

See http://www.TheOncologist.com for supplemental material available online. 